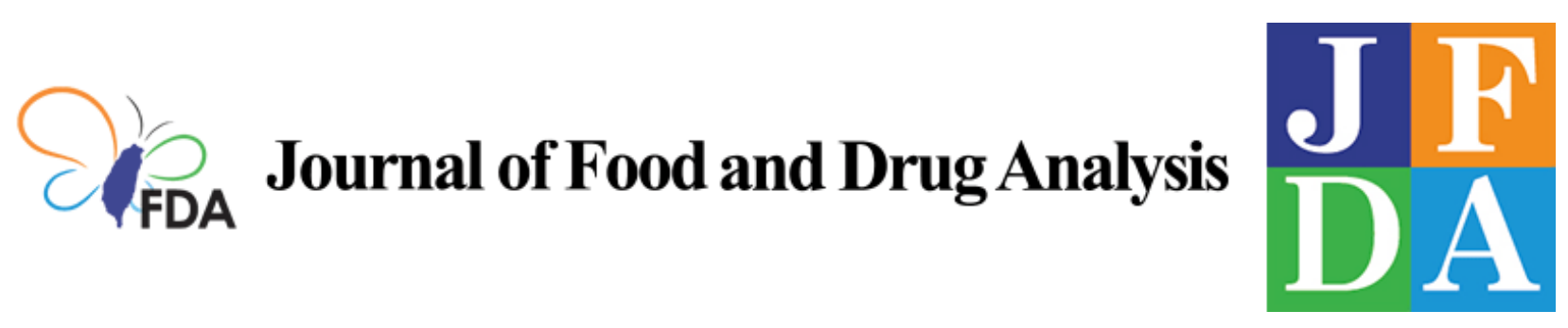

Volume 29 | Issue 1

Article 13

2021

\title{
New Psychoactive Substances in Taiwan: the Current Situation and Initiative for Rational Scheduling
}

Follow this and additional works at: https://www.jfda-online.com/journal

Part of the Medicinal Chemistry and Pharmaceutics Commons, Pharmacology Commons, Substance Abuse and Addiction Commons, and the Toxicology Commons

(c) (i) (9)

This work is licensed under a Creative Commons Attribution-Noncommercial-No Derivative Works 4.0 License.

\section{Recommended Citation}

Yu, Wen-Jing; Cottler, Linda B.; and Li, Jih-Heng (2021) "New Psychoactive Substances in Taiwan: the Current Situation and Initiative for Rational Scheduling," Journal of Food and Drug Analysis: Vol. 29 : Iss. 1 , Article 13.

Available at: https://doi.org/10.38212/2224-6614.3225

This Original Article is brought to you for free and open access by Journal of Food and Drug Analysis. It has been accepted for inclusion in Journal of Food and Drug Analysis by an authorized editor of Journal of Food and Drug Analysis. 


\title{
New psychoactive substances in Taiwan: The current situation and initiative for rational scheduling
}

\author{
Wen-Jing Y ${ }^{1,2}$, Linda Cottler ${ }^{3}$, Jih-Heng $\operatorname{Li}^{1,4,5, *}$ \\ ${ }^{1}$ Doctoral Degree Program in Toxicology, College of Pharmacy, Kaohsiung Medical University, Kaohsiung, Taiwan \\ ${ }^{2}$ Food and Drug Administration, Ministry of Health and Welfare, Taiwan \\ ${ }^{3}$ College of Public Health and Health Professions and College of Medicine, University of Florida, Gainesville, FL, USA \\ ${ }^{4}$ Master Degree Program in Toxicology and School of Pharmacy, College of Pharmacy, Kaohsiung Medical University, Taiwan \\ ${ }^{5}$ Department of Medical Research, Kaohsiung, Medical University Hospital, Kaohsiung, Taiwan
}

\begin{abstract}
Use of New Psychoactive Substances (NPS) has posed a global threat to public health and the security of the population. As of December 2019, the NPS items identified in total have outnumbered by three to one the controlled substances listed in the 1961 and 1971 UN Drug Conventions. However, most of these NPS have not been scheduled by the United Nations because of their easy modification on the chemical structures to shun control. Currently, the scheduling and control of NPS is mostly at the national level and a rational scheduling of NPS by objective assessments is essential but often lacking. To rationally schedule NPS, the NPS misuse situation was firstly estimated with the Taiwanese Substance Misuse Monitoring and Reporting Systems (SMMRS) from 2006 through 2019. Then, the assessment of drug-related harms with an expert Delphi procedure for drug scheduling was performed. The epidemiological analysis revealed that among 37 substances commonly misused in Taiwan, heroin posed the highest risk, followed by (meth)amphetamine and ketamine. Of note, misuse of NPS, such as ketamine, synthetic cannabinoids (JWHs, AM-2201, XJR-11), synthetic cathinones (MDPV, bkMDMA, 4- MMC etc.), phenethylamines (PMMA, FMA, 2C-B, 2C-E etc.), piperazines (BZP, TFMPP) and tryptamines (5$\mathrm{MeO}-\mathrm{DIPT}$ ) has been on the rise. Though perceived drug-related harms differed among experts with different professional backgrounds, the differences were not significant. Four dimensions of drug-related harms- addiction, misuse, social harm and physical harm- integrated from Nutt's model and scheduling criteria of Taiwan's Statute for the Prevention and Control of Illicit Drugs (SPCID), were further divided into 11 indicators and applied to assess harms of the 37 substances. Among the 11 indicators that corresponded to the four dimensions, 7 had significant prediction capabilities. Additionally, prevalence of misuse nationally was an important predictor of harm assessment. These indicators of harm assessment of drug misuse can help develop a proper scheduling system for the management of controlled/illicit drugs. In conclusions, drug scheduling is the first step toward proper management of drug use problems. Facing the threats of NPS, it is imperative to implement a rational and effective scheduling system for appropriate management. This study provides a mechanism to scrutinize, and improve, the current evaluation process for NPS scheduling.
\end{abstract}

Keywords: Drug scheduling, Misuse trend, New psychoactive substances (NPS), Taiwan

\section{Introduction}

T $\mathrm{n}$ the $20^{\text {th }}$ Century, the enactment of three 1 United Nations Drug Conventions (the 1961 Single Convention on Narcotic Drugs, the 1971 UN Convention on Psychotropic Substances and the 1988 UN Convention against Illicit Traffic in Narcotic Drugs and Psychotropic Substances) has been the foundation up to the present day international drug control system. However, in recent years, use of new psychoactive substances (NPS) has emerged as a new threat to public health and social security because of the elusiveness of this use from the UN Drug Conventions and the drugs' uncertain toxicological profiles [1, 2]. NPSs are defined by the United Nations Office on Drugs and Crime (UNODC) as substances that are not controlled under the 1961 or 1971 UN Drug Conventions and predominantly derivatives or analogues of existing controlled substances $[3$,

Received 25 September 2020; revised 26 January 2021; accepted 2 January 2021.

Available online 15 march 2021

* Corresponding author at: Master Degree Program in Toxicology and School of Pharmacy, College of Pharmacy, Kaohsiung Medical University, 100 ShihChuan $1^{\text {st }}$ Road, San-Min District, Kaohsiung 80708, Taiwan.

E-mail address: jhlitox@kmu.edu.tw (J.-H. Li). 
4]. They are classified into 9 categories, including synthetic cannabinoids, synthetic cathinones, ketamine and PCP-type substances, phenethylamines, piperazines, tryptamines, aminoindanes, plant-based substances and others [5]. As of December 2019, The UNODC had identified a total of 950 NPS, a number that is more than 3 times the 273 substances listed in the 1961 and 1971 conventions [6].

Owing to the lack of an international consensus on the legislative control of NPS, at present, scheduling of NPS mainly depends on the national legislation and drug policy. In Taiwan, NPS are also scheduled as controlled substances according to the national drug-related laws that were enacted primarily for the control of known traditional addictive (dependence-inducing) drugs. Our previous study on the comparison of the legislative mechanism for NPS control between Taiwan, South Korea and Japan indicated that rigid criteria-addictive potential, abuse liability and social harm liability - for scheduling NPS as controlled substances may explain why Taiwan controlled the fewest NPS items [7]. Therefore, for effective NPS control in Taiwan, timely and flexible legislative mechanisms with a sensitive monitoring system and quick response to comprehensive scientific evidence about the rapidly emerging NPS are essential [7].

Nutt et al. have demonstrated the importance of rational scheduling for drug control using a ninecategory of harm model with an expert Delphi procedure [8]. This model evaluated drug-related harms with nine parameters grouped into three dimensions-physical harm, dependence and social harms- to develop a rational scale for assessment of the harm of drugs. However, the model did not take individual drug misuse into consideration. Take Taiwan for example- the misuse of ketamine and amphetamine(s) has been more prevalent than that of cocaine and street methadone, but it would not be counted as a contributing factor of misuse liability. In other words, the nine-category of harm model did not fully correspond to the legislative criteria of addictive potential, misuse liability and social harm liability to schedule controlled substance in Taiwan.

In Taiwan, misuse of club drugs, including MDMA, ketamine, flunitrazepam, and zolpidem, revealed a new pattern of drug use since the early 2000s as determined by ketamine-positive urine samples and increased ketamine seizures [9]. The harms caused by ketamine, including dependence, cognitive impairment, emergence phenomenon, and interstitial cystitis, may be greater than expected $[8,10]$. Ketamine has been placed as a schedule III drug in Taiwan since 2002. Because of its predominant abuse and toxicological effects, rescheduling of ketamine to schedule I or II has been debated in recent years. Besides ketamine, the misuse of some other NPS, such as cathinones and synthetic cannabinoids has also been a new concern $[9,11]$. However, some NPS are yet to be controlled due to the rigid criteria of drug scheduling.

As noted, national laws control appropriate NPS scheduling. In this study, the three legislative criteria of addictive potential, misuse liability and social harm liability were taken into consideration to determine indicators of drug-related harms for assessment beginning with misuse in Taiwan. The epidemiological data of drug seizure and use were collected from "Drug abuse cases and test statistics data" [12] and weighted as an indicator for misuse liability. Then, Nutt's nine-category matrix of harm model was modified to compare the drug-related harms with and without consideration of the epidemiological data on misuse. Using these criteria, we present an objective and comprehensive evaluation of the potential of NPS-related harms for appropriately suitable scheduling.

\section{Materials and Methods}

For rational scheduling of NPS, NPS misuse data was first evaluated using epidemiological approaches. The misuse data of major traditional drugs served as a reference for comparison. Then, the assessment of drug-related harms with an expert Delphi procedure for drug scheduling was conducted.

\subsection{Data collection}

Drug misuse data representing both the supply and demand sides was obtained from the Substance Misuse Monitoring and Reporting Systems (SMMRS), maintained on a monthly basis by the Taiwan Food and Drug Administration (TFDA) of the Ministry of Health and Welfare (MOHW). The subsystems of SMMRS briefly described below [12].

\subsubsection{Data collection subsystem on arrests and drug seizures (SADS)}

The data, collected monthly, was from the Investigation Bureau, Ministry of Justice; the National Police Administration, Ministry of Interior; and the Headquarters of Military Police, Ministry of Defense; data used was from 2006 through 2019. 


\subsubsection{Data collection subsystem on laboratory drug testing of urine (LDTU) for court referrals}

The data collected here included sources just described with the addition of all local health departments.

\subsubsection{Reporting subsystem for addiction treatment (RSAT)}

Data was collected from the MOHW-designated 150 psychiatric hospitals for addiction treatment from 2006 through 2019. In this subsystem, cases of substance misuse were reported online.

\subsection{Evaluation of drug-related harms with an expert Delphi procedure}

The evaluation of harm using an expert Delphi procedure was conducted with a modification of the procedure outlined by Nutt et al. [8]. Harms of drugs were evaluated with each expert providing scores separated into three dimensions: physical harm, dependence and social harms. Each dimension was further divided into three aspects of harm. The dimension of physical harm consisted of acute, chronic and intravenous harm; the dimension of dependence included intensity of pleasure, psychological dependence and physical dependence; and the dimension of social harms included intoxication, other social harms and health care costs.

Nutt's nine indicators of harm did not include epidemiological misuse-related outcomes. In Taiwan, according to the mandate of the Statute for the Prevention and Control of Illicit Drugs (SPCID), a substance can only be scheduled as a controlled drug if it possesses all three characteristics: addictive potential, misuse liability and social harm liability, defined as follows:

(1) Addictive potential: euphoria, withdrawal and psychological dependence.

(2) Misuse liability: international misuse prevalence, national misuse prevalence and different regulations among different countries.

(3) Social harm liability: infectious hazard, criminality and poly-drug use hazard.

This SPCID-based scheduling system, on the other hand, did not factor physiological harm into account. Therefore, this study tried to incorporate the harmbased approach of Nutt et al. and the SPCID-based scheduling principles of addictive potential, misuse liability and social harm liability by adding the fourth dimension-physical harm. For the Delphi survey, these 4 dimensions of harm were further divided into 11 indicators as shown in Table 1: euphoria, withdrawal, pharmacokinetics, psychological dependence, international prevalence, national prevalence, criminality (violent attacks, accidents, etc.), social costs (work loss, medical costs), acute physical hazards, chronic physical hazards, and infection hazards (communicable disease transmission).

Twenty-three experts in the fields of biomedical (addiction and physical harm) and social sciences (misuse and social hazard) were invited to participate in the risk assessment of 37 commonly used drugs/substances (15 traditional substances, 18 NPS and 4 non-scheduled substances). The survey was focused on the harm scored with 11 indicators on these 37 addictive substances most often used improperly. The expert opinion was carried out in two stages. In the first stage, we provided the definitions of assessment indicator and questionnaire for experts to fill in the substance hazard assessment scores. In the second stage, in addition to the results of the survey obtained from the opinions of experts in the first stage, we also provided the participating experts reference information such as the legal classification and misuse situations of relevant substances in the UN, USA, UK, Canada, Singapore and Taiwan, as well as toxicological profiles of these substances. There were 19 experts participated in the first stage and 18 in the second stage.

\subsection{Data analysis}

Statistical analysis was performed with SPSS version 20.0. Data was tested by $t$ test, pared-t test, one way ANOVA, and multiple linear regression with a $p$-value $<0.05$ determining a statistically significant difference.

To determine the accuracy of the prediction model, the root mean squared error (RMSE) was applied [13, 14]. The RMSE is a standard method to measure the error of a model in predicting quantitative data. In this study, it serves as an estimator for the standard deviation $\sigma$ of the distribution of the errors from our model's prediction.

\section{Results}

\subsection{Analysis of drug misuse trends in Taiwan}

\subsubsection{The supply side}

\subsubsection{Seizures of major illegal drugs.}

According to the data of SADS, the confiscated amounts of ketamine, a major NPS category, prevailed all drug seizures from 2006 to 2019 in Taiwan. There were three peaks of ketamine seizures in 
Table 1. Indicators of three different models for evaluation of drug-related harms.

\begin{tabular}{|c|c|c|c|c|}
\hline \multicolumn{2}{|c|}{ Parameters of Nutt's model } & \multirow[t]{2}{*}{ Indicators based on SPCID } & \multicolumn{2}{|c|}{ Integrated Indicators in this study } \\
\hline Category & Parameter & & Category & Indicator \\
\hline \multirow[t]{3}{*}{ Dependence } & Intensity of pleasure & Addiction & Addiction & Euphoria \\
\hline & Physical dependence & & & Withdrawal \\
\hline & Psychological dependence & & & Psychological dependence \\
\hline \multirow[t]{5}{*}{ Social harm } & Intoxication & & & Pharmacokinetics \\
\hline & - & Misuse & Misuse & International misuse prevalence \\
\hline & - & & & National misuse prevalence \\
\hline & Other Social harms & Social harm & Social harm & Criminality \\
\hline & Health-care costs & & & Health-care costs \\
\hline \multirow[t]{3}{*}{ Physical harm } & Acute & & Physical harm & Acute \\
\hline & Chronic & - & & Chronic \\
\hline & Intravenous harm & & & Infectious hazard \\
\hline
\end{tabular}

2010, 2014 and 2019, and each peak was much higher than the previous one (growth rate $>26 \%$ ). A record high of $3,303 \mathrm{Kg}$ of ketamine was seized in 2014 and then gradually decreased yearly, though still greater than 1,000 kg/year after 2016 (Fig. 1). Ketamine was the most highly seized drug at 1,599.7 $\mathrm{Kg} /$ year, followed by (meth) amphetamine $399.0 \mathrm{Kg} /$ year, heroin $146.5 \mathrm{Kg} /$ year and cannabis $66.0 \mathrm{Kg}$ / year. The seizure of (meth)amphetamine, a major misused drug in the 1990s in Taiwan, resurged in recent years and in one year (2018) exceeded ketamine seizures. Overall, ketamine and (meth) amphetamine were the most seized drugs in Taiwan over this period of time, followed by heroin, cannabis and nimetazepam (Fig. 1-A).

\subsubsection{Seizures of NPS other than ketamine.}

Seizures of large quantities of ketamine over the years indicated the NPS issue deserves attention (Fig. 1-B). Besides ketamine, other NPS, mainly synthetic cathinones, synthetic cannabinoids and phenethylamines, have also been seized. The results showed that after XLR-11, a synthetic cannabinoid, was regulated as a schedule III drug in 2014, its seizure in 2015 was significantly reduced to none. Synthetic cathinones, such as MDPV and bkMDMA, also showed seizures decreased significantly after regulation (Fig. 1-B).

Para-methoxymethamphetamin (PMMA), a phenethylamine-type NPS, has been controlled as a schedule II drug and was first seized in 2009 at 30.2 grams, and except for a seizure of 960.1 grams in 2015, there were no further seizures. In 2015, methoxymethcathinone was regulated as a schedule II drug. Except for 3,240.8 grams seized at the same year, there were no further seizures.

In contrast, seizures of some NPS continued to increase significantly after regulation. Synthetic cathinones such as mephedrone and bk-MDMA were continuously seized each year after being regulated from 2014 to 2019 as shown in Fig. 1-B.

\subsubsection{Laboratory drug testing of non-urine (confiscated} chemical) samples.

Results of drug testing on non-urine samples elucidate which substances have been possessed or sold. According to the total cases of positive substances detected from 2006 to 2019, the main substances in order were: (meth)amphetamine, ketamine, heroin, nimetazepam, mephedrone, methyl- $\alpha$-ethylaminopentiophenone (MEAPP), bk-MDMA, MDMA, XLR-11, and methoxymethamphetamine. In addition to ketamine, the average annual positive cases of some NPS, such as mephedrone, MEAPP and bk-MDMA were all more than 3,000 cases/year; XLR-11 was more than 1,000 cases/year and methoxymethamphetamine 423 cases/year, revealing that NPS problems deserve our attention (Fig. 2-A). Of note, multi-component substances detected in non-urine samples have become more prevalent in recent years.

The results of positive cases detected in non-urine samples can also be approached by category as follows:

(1) Phenylethylamines: (Meth)amphetamine was the most predominant substance, followed by MDMA and PMMA. The samples detected with phenylethylamine mainly contained a single component, but multiple components have been detected in recent years. The cases of methoxymethamphetamine (PMMA) decreased yearly since its regulation in 2006 except for a transient increase in 2007.

(2) Ketamine: Cases of ketamine gradually increased from 2006 to 2015 with a peak of over 30,000 cases yearly in 2014 and 2015, and then decreased from 2016 to 2019 although still maintained over 20,000 cases per year. 
A

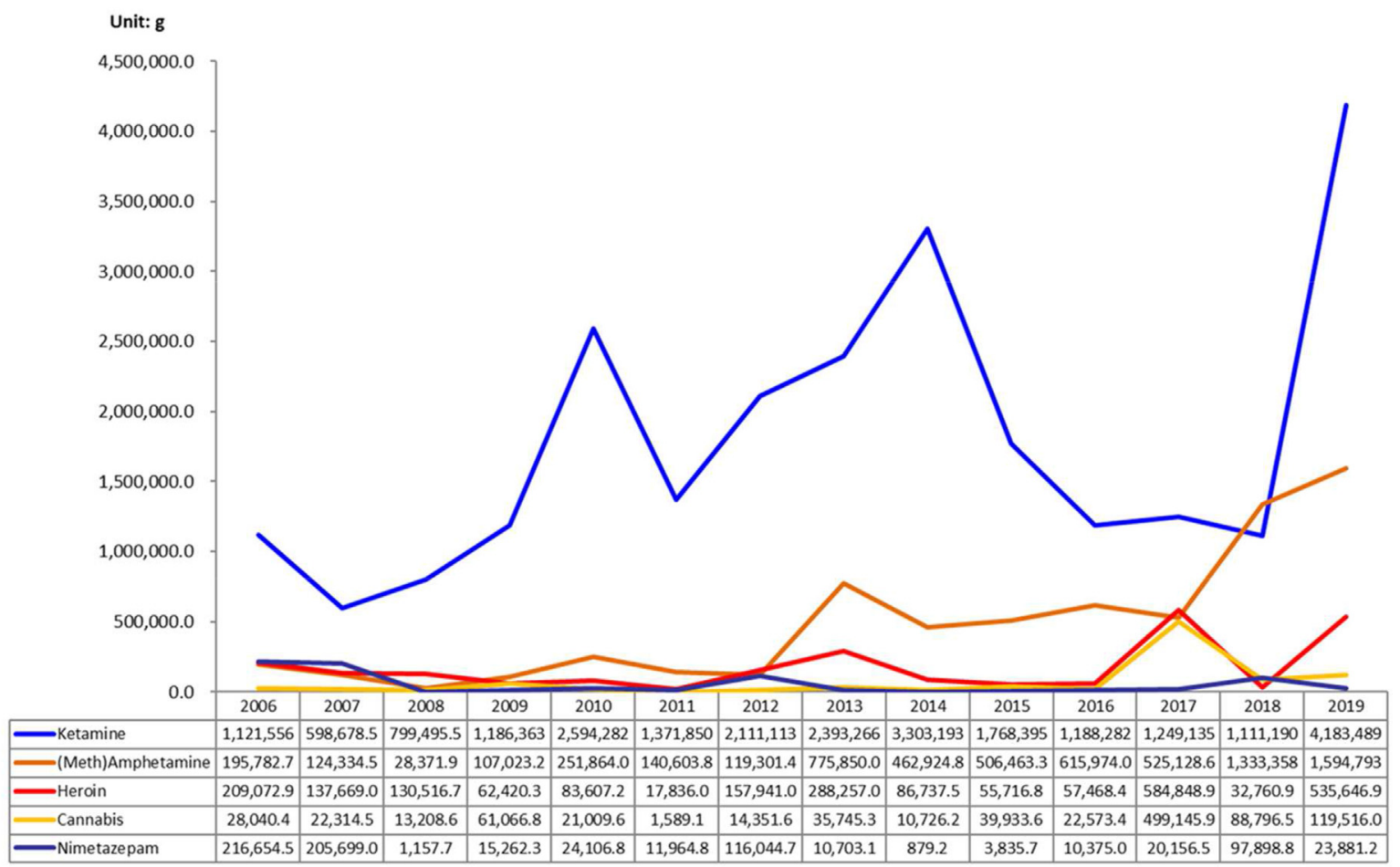

B

Unit: g $\quad 35000$

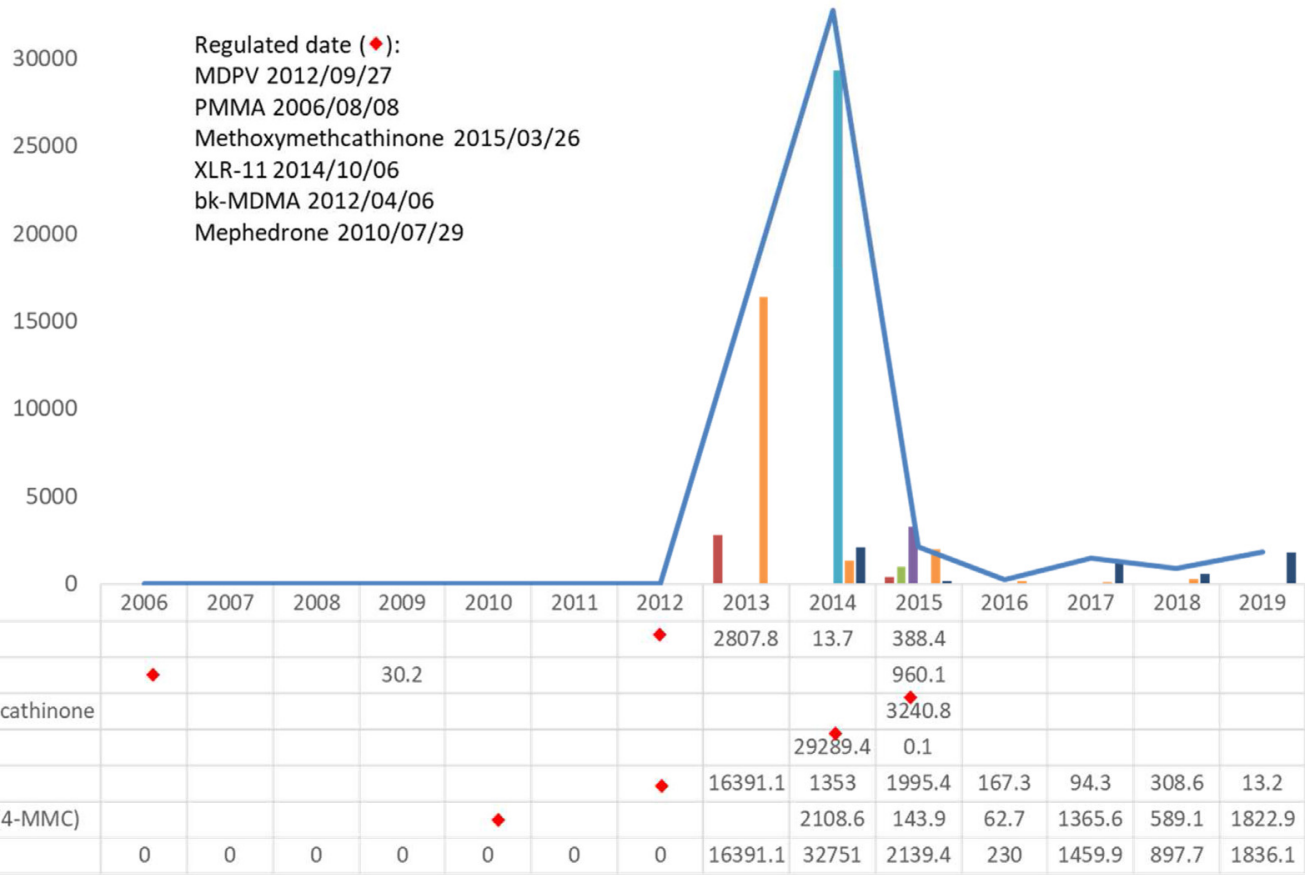

Fig. 1. Seizures of (A) major illegal drugs (B) illegal NPS other than ketamine in Taiwan from 2006 through 2019. 
A

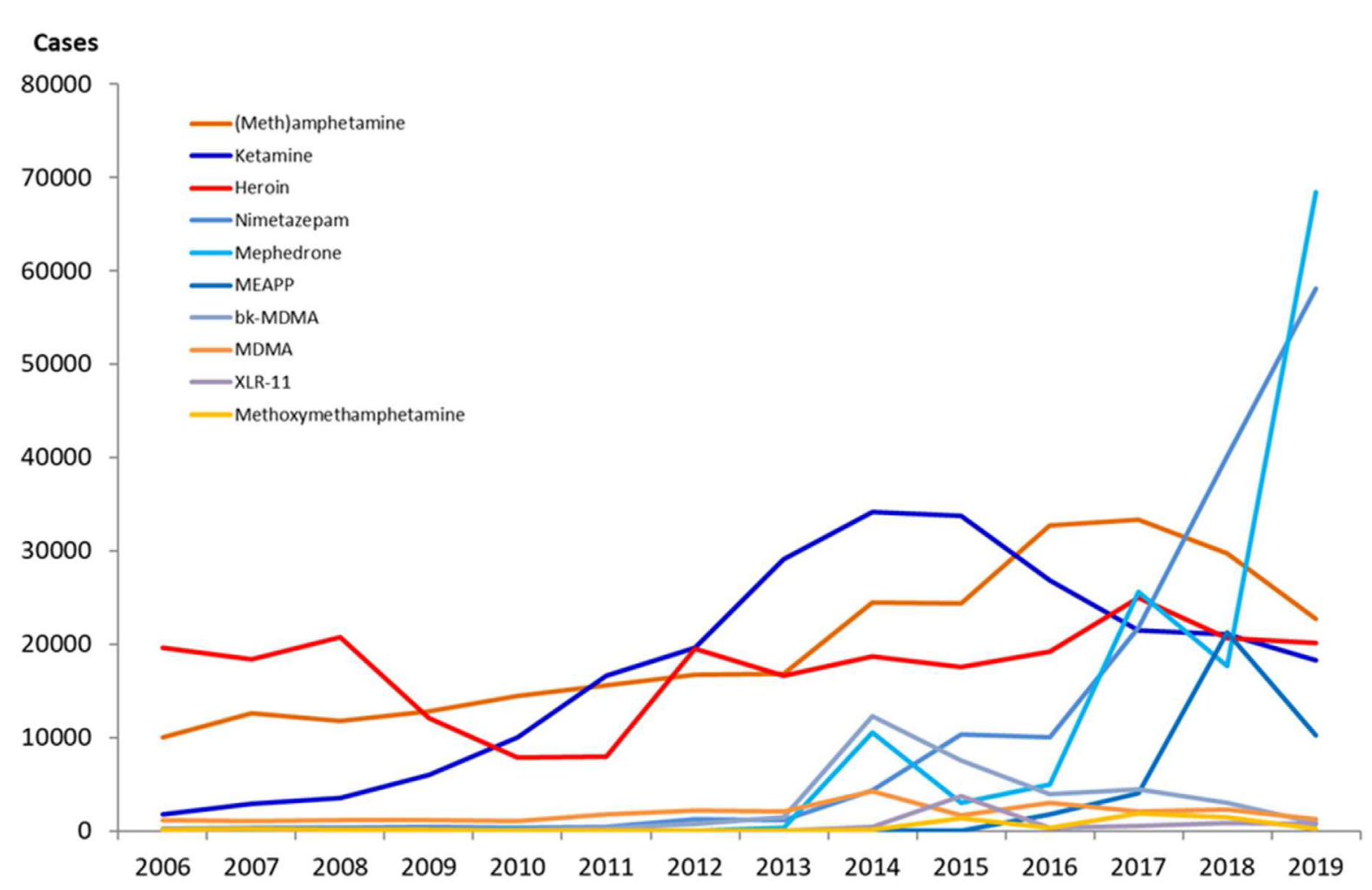

B

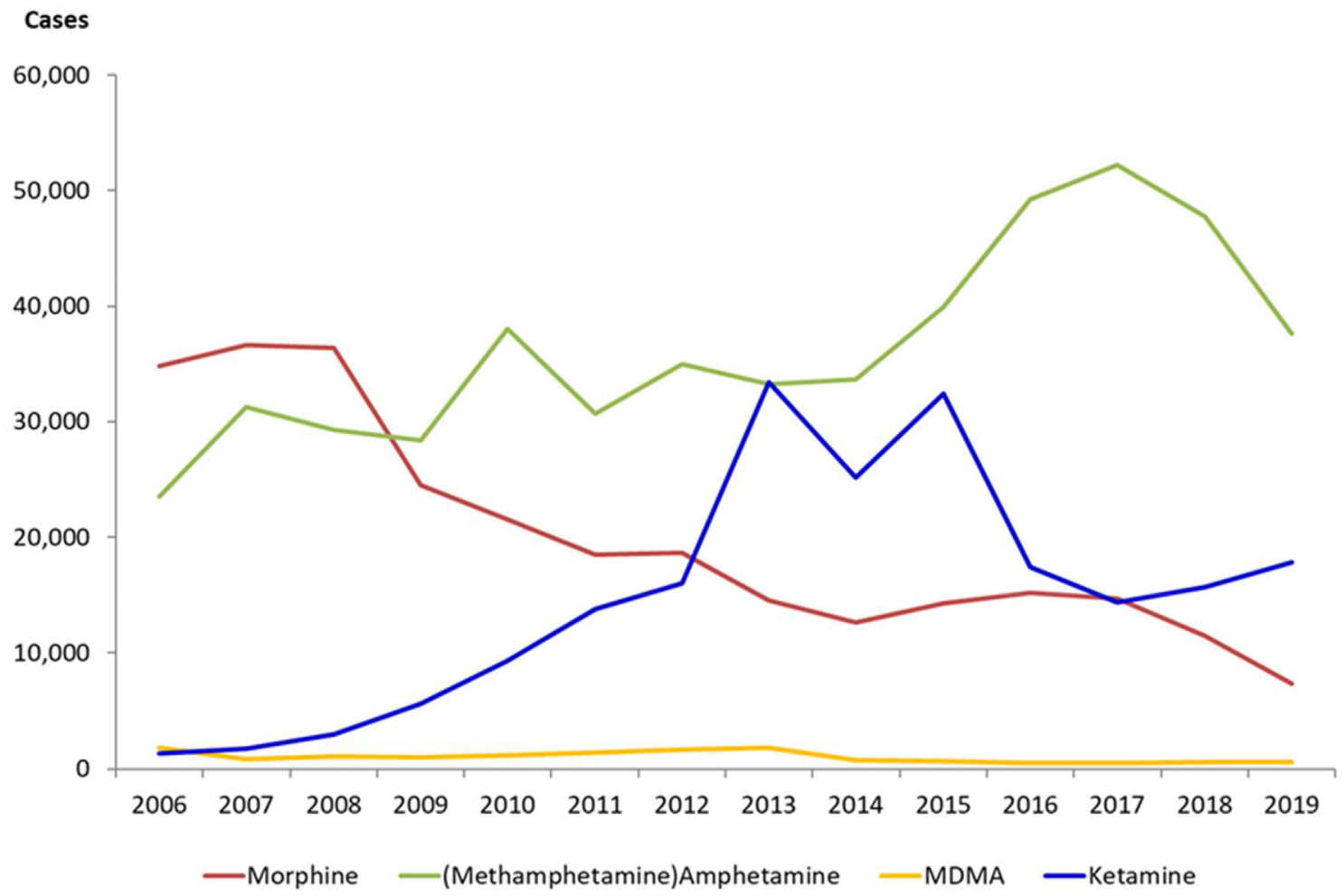

Fig. 2. Positive drug testing cases of (A) non-urine samples and (B) urine samples in Taiwan. 
(3) Opioids: Cases detected with heroin as the single component fluctuated around 20,000 per year. Sporadic cases of acetylcodeine and acetylmorphine, which have not been regulated, were also reported (data not shown). These two unregulated items were mainly detected in cases with multiple drugs.

(4) Cathinones: Since 2009, several synthetic cathinones, including mephedrone, MEAPP and bkMDMA, have been detected. They were mainly detected with multiple drugs. Among them, the number of positive cases of mephedrone (schedule III) increased sharply from 2015 to 2019. MEAPP was regulated in 2017. The number of MEAPP positive cases increased from 2016 to 2018, then decreased in 2019. bk-MDMA was regulated in 2012. The number of bkMDMA positive cases increased from 2012 to2015 yearly, then dropped significantly in 2016.

(5) Cannabis: Cases of cannabis without other drugs were mainly detected from 2006 to 2011. In 2012, about half of cases with cannabis and other drugs were detected. Then the number of positive samples containing cannabis and multiple components increased annually. Among them, XLR-11 was regulated in 2014. In 2014, the number of XLR-11 positive cases was 411 . Then in 2015 , the number of positive cases increased significantly to 3,689 and the number of positive cases was also detected every year.

(6) Tryptamines: Cases of tryptamines were mainly detected in samples with multiple drugs. 5MeO-MIPT (schedule III) was the major tryptamine-type NPS. The number of 5-MeO-MIPT positive cases increased significantly in 2015 and 2017. 5-MeO -DIPT (schedule IV) was also detected sporadically.

(7) Piperazines: Cases of piperazines were mainly detected with multiple drugs. Trifluoromethylphenylpiperazine (schedule III) was the most commonly detected, followed by N-Benzylpiperazine (schedule II). Both positive cases detected in 2014 were significantly on the rise since.

\subsubsection{The Demand side}

Based on the results of "2014 National Substance Abuse Survey", the prevalence of illegal drugs among 12 to 64 ages in Taiwan was $1.29 \%$. The most commonly misused illegal drugs were in the following order: amphetamine(s) $(0.60 \%)$, ketamine $(0.39 \%)$ and cannabis $(0.30 \%)$ [15].

\subsubsection{Drug addiction treatment.}

Data on drug mentions among Treatment Admissions indicate that heroin and methamphetamine remained the two predominant drug problems from 1999 to 2019, heroin increased from 902 mentions in 1999 to 16,891 mentions in 2019 and methamphetamine increased from 1,120 mentions in 1999 to 13,038 mentions in 2019; while ketamine-dependent admissions increased from 99 mentions in 2006 to 1,760 mentions in 2019. From 2006 to 2019 , ketamine increased from $0.6 \%$ to $5.1 \%$ (Fig. 3). Other than ketamine, the treatment mentions related to NPS misuse have only been reported since 2014.

In 2019 , heroin $(49.4 \%)$ and (meth)amphetamine $(38.0 \%)$ remained the two most prevalent drugs in the mentions of treatment admissions, followed by, ketamine $(5.1 \%), \operatorname{MDMA}(2.3 \%)$ and cannabis $(1.7 \%)$. In treatment mentions of NPS other than ketamine, FMA $(51.7 \%)$ was the most prevalent drug, follow by MDPV (10.0\%), CMA (10.0\%), Mephedrone $(8.3 \%)$, Thiamylal $(5.0 \%)$ and $\mathrm{K} 2(5.0 \%)$ as shown in Fig. 3.

\subsubsection{Urine drug testing.}

(Meth)amphetamine, heroin, ketamine and MDMA were found among results of urine tests in persons suspected of using drugs, as shown in Fig. 2-B with average annual positive cases of: (meth) amphetamine 36,329, heroin 21,056, Ketamine 14,560 and MDMA 1,052. Ketamine positive cases were much more than many schedule II drugs such as: MDMA, morphine-N-oxide (615 cases/year), codeine $(\geq 5 \%)$ (232 cases/year), and cannabis (211 cases/Year).

Since ketamine was placed as a schedule III drug in 2002 [10], the positive cases have increased substantially from 2006 to 2013. Although there was a transient decrease from 2015 to 2017, ketamine positive cases increased again from 2018 to 2019. Since 2012, the number of ketamine positive cases was even higher than that of morphine positive cases, which were interpreted as heroin use after metabolism.

While the majority of positive urine samples identified were single drugs, cases with multiple drugs increased year by year. Specifically, eightyeight single substances were detected in urine samples from 560,096 people testing positive, while 2,310 multiple substances were identified from 300,294 positive cases in urine samples from 2006 to 2019. The detection of single substance accounted for $65 \%$ of the total number of positive samples, and the multiple substances accounted for $35 \%$. Besides ketamine, other NPS included: synthetic cathinones (bk-MDMA, bk-MBDB, bk-MBDB, bk-DMBDB, Ethylone, CMC, CEC, Cl-Alpha-PVP); and phenethylamines (2C-E).

As of December 2019, all regions/countries around the world have reported 950 items of NPS to the 

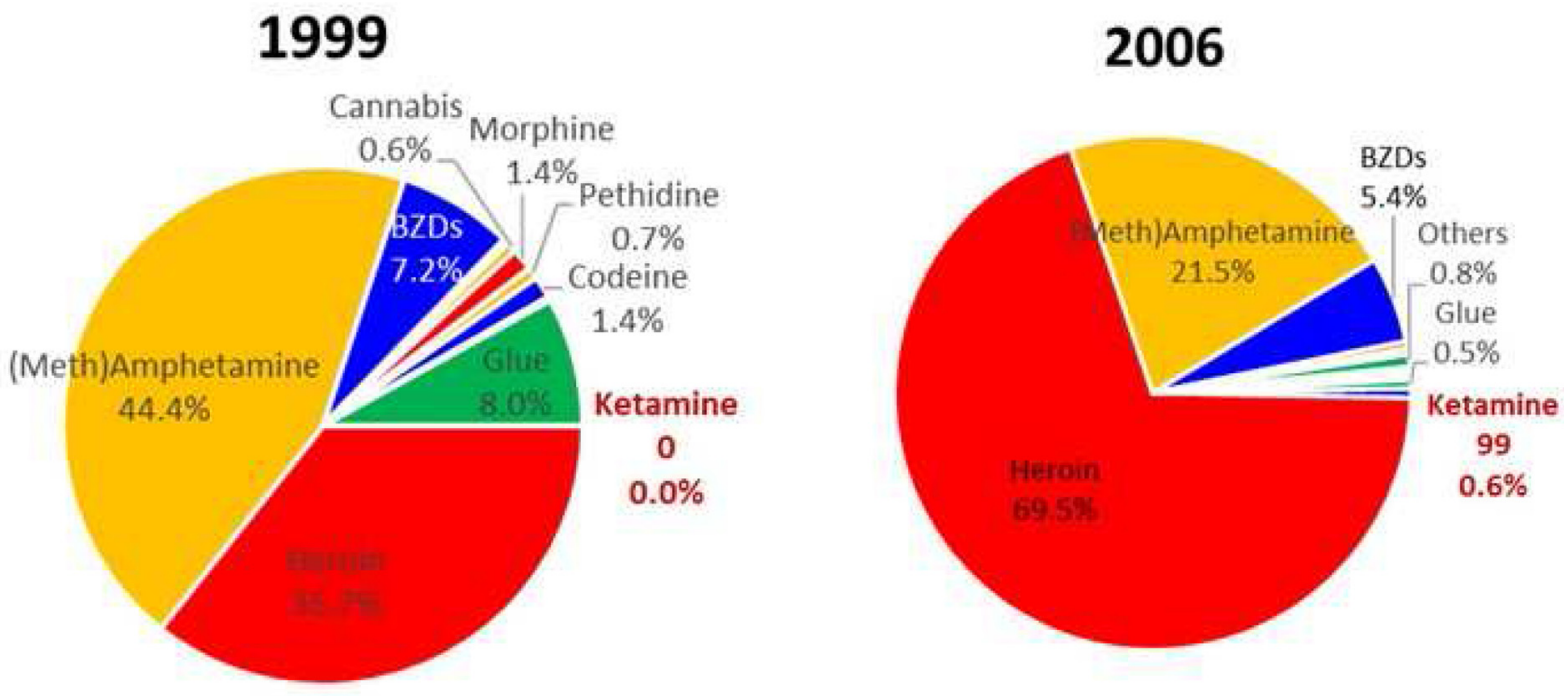

\section{4}
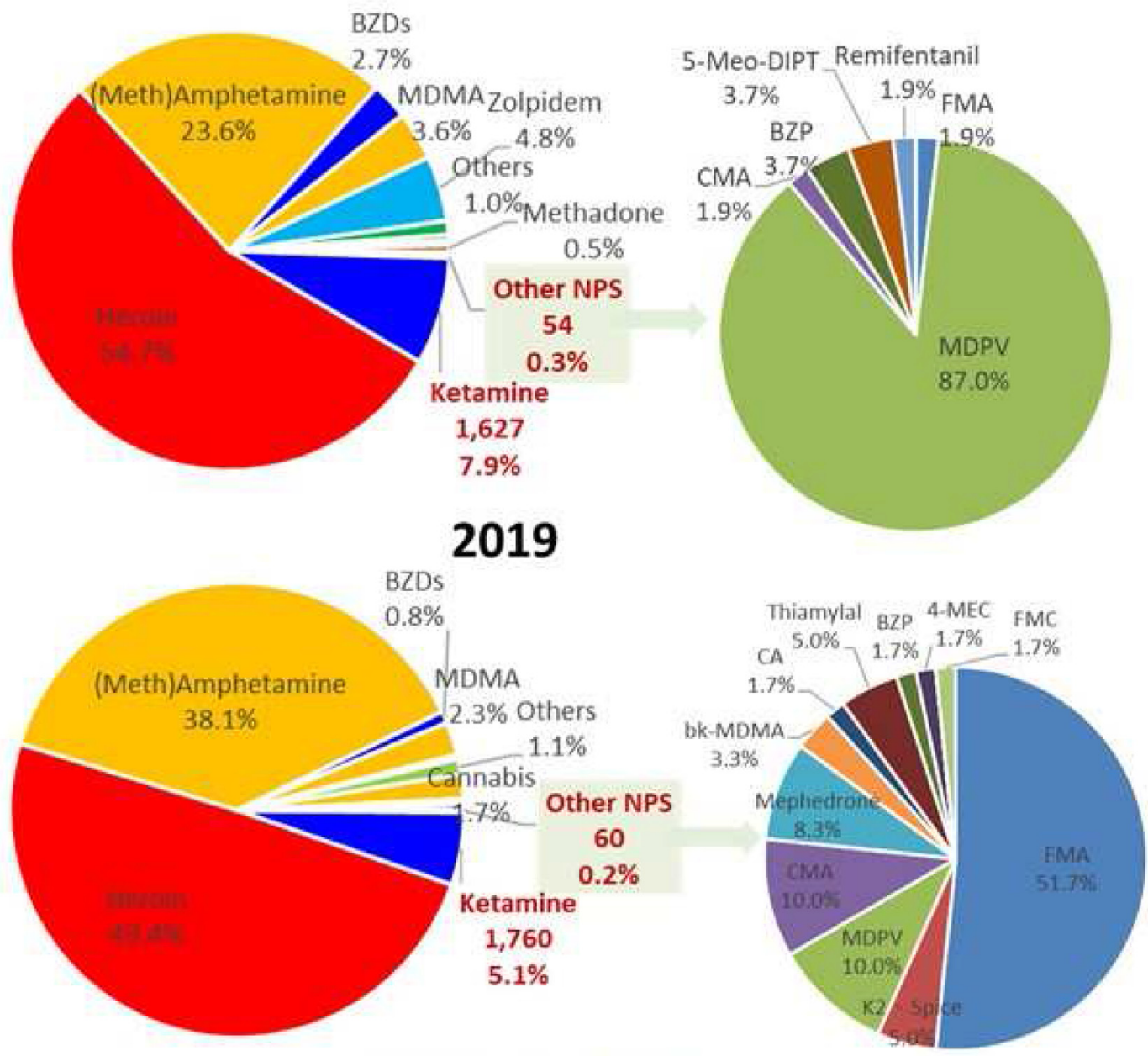

\section{Class: $\square 1^{\text {st }} 2^{\text {nd }} \square 3^{\text {rd }} 4^{\text {th }} \square$ none}

Fig. 3. Proportions of Different Drug Mentions among Treatment Admissions to Hospitals in Taiwan from 1999 through 2019. 
United Nations Office on Drugs and Crime (UNODC). By contrast, in Taiwan, by the end of 2019, there were only 52 NPS items scheduled. These 52 NPS items are described by the category as follows:

(1) Synthetic cannabinoids: 9 schedule III drugs (JWH-018, JWH-073, JWH-250, HU-210, CP47,497, JWH-122, AM-2201, XLR-11 and ABPINACA).

(2) Synthetic cathinones: 3 schedule II drugs (MDPV, methoxymethcathinone and Alpha-PVP); 17 schedule III drugs (mephedrone, methylone, 4methylethcathinone, fluoromethcathinone, 3,4methylenedioxy-N-ethylcathinone, chloromethcathinone, bromomethcathinone, butylone, methylbutylone, methylpentedrone, chloroethcathinone, methyl-N-benzylcathinone, ClAlpha-PVP, ethylmethcathinone, Chloro- $\alpha$-PPP, ethylethcathinone and methyl-N,N-dimethylcathinone) and 1 schedule IV drug (chlorodimethylcathinone).

(3) Ketamine and PCP-type substances: 3 schedule III drugs (Ketamine, methoxetamine and fluoroketamine).

(4) Phenethylamines: 6 schedule II drugs [N,NDimethylamphetamine, p-methoxymethamphetamine, fluoromethamphetamine, chloromethamphetamine, lisdexamphetamine and (2-methylaminopropyl)benzofuran]; 6 schedule III drugs (4-bromo-2,5-dimethoxyphenethylamine, p-methoxyethylamphetamine, chloroamphetamine, 25B-NBOMe, fluoroamphetamine and 2,5dimethoxyphenethylamine).

(5) Tryptamines: 1 schedule III drug (5-MeO-MIPT); 1 schedule IV drug (5-MeO-DIPT)

(6) Piperazines: 1 schedule II drug (1-benzylpiperazine); 1 schedule III drug [1-(3trifluoromethylphenyl)].

(7) Plant-based substances: 2 schedule II drugs (peyote and khat). 1 schedule III drug (kratom).

Most of the regulated items were schedule II and schedule III substances. The NPS items regulated as schedule II were mostly amphetamine isomers or analogues. The schedule III of NPS were mainly synthetic cathinones and synthetic cannabinoids.

\subsection{Assessment of drug-related harms with an expert Delphi procedure for drug scheduling}

\subsubsection{Evaluation of harm scores}

The ranking of substances of misuse by harm scores based on 9 assessment parameters of Nutt's research is shown in Fig. 4-A; 8 indicators based on
Taiwan's Statute are shown in Fig. 4-B and 11 assessment parameters of this research are shown in Fig. 4-C. There were significant differences between the 11 evaluation indicators in this study, the 8 indicators based on Taiwan's SPCID and the 9 assessment parameters obtained by the Nutt study ( $p$-value $=0.000<0.05$ by $t$ test).

The result of ranking according to harm assessment scores of 37 common misuse substances is shown in Fig. 4. The top ten substances based on Nutt's 9 parameters were heroin, cocaine, amphetamine, fentanyl, 2C series, MDMA, alcohol, MDPV, PMMA and 25B-NBOMe; the top ten based on 8 indicators on Taiwan's SCPID were: heroin, amphetamine, cocaine, fentanyl, alcohol, MDMA, 2C series, MDPV, ketamine and Oxycodone; and based on the 11 indicators in the integrated study were: heroin, amphetamine, cocaine, fentanyl, alcohol, MDMA, 2C series, MDPV, ketamine and PMMA. While heroin was first on each list, cocaine, amphetamine and fentanyl were 2 through 4 on each list, though in different order.

According to Fig. 4, we found the ranking of traditional drugs by the harm scores to have been affected by the indicator of misuse prevalence. Specifically in the United Kingdom, where cocaine misuse was more serious, the harm score of cocaine ranked second whereas in Taiwan, amphetamine ranked second because of its perceived severity.

The harm score ranking of NPS varied in these 3 models. The top 5 by Nutt's 9 parameters were $2 \mathrm{C}$ series, MDPV, PMMA, 25-NBOMe and BZP; by 8 indicators based on Taiwan's SPCID were $2 \mathrm{C}$ series, MDPV, ketamine, BZP and Trifluoromethylphenylpiperazine (TFMPP); by 11 indicators in this study were 2C series, MDPV, ketamine, PMMA and 25-NBOMe.

Results from the experts indicated that the harm caused by certain NPS was greater than many other schedule II controlled drugs, such as cannabis, GHB and methadone. Although alcohol was not a controlled drug, experts gave it higher harm scores than MDMA, cannabis, GHB, etc. The harm scores rank is shown in Fig. 4.

\subsubsection{Mean harm scores between 2 stages}

After reviewing the harm assessment scores of the individual substances, a comparison of the harm scores between stage 1 and stage 2 was performed. The results indicate they were not statistically different (Fig. $5, \mathrm{p}$-value $>0.05$ by $\mathrm{t}$ test).

\subsubsection{The prediction model analysis}

Since there was no significant difference between the two stages, we used the harm scores of stage 2 to 

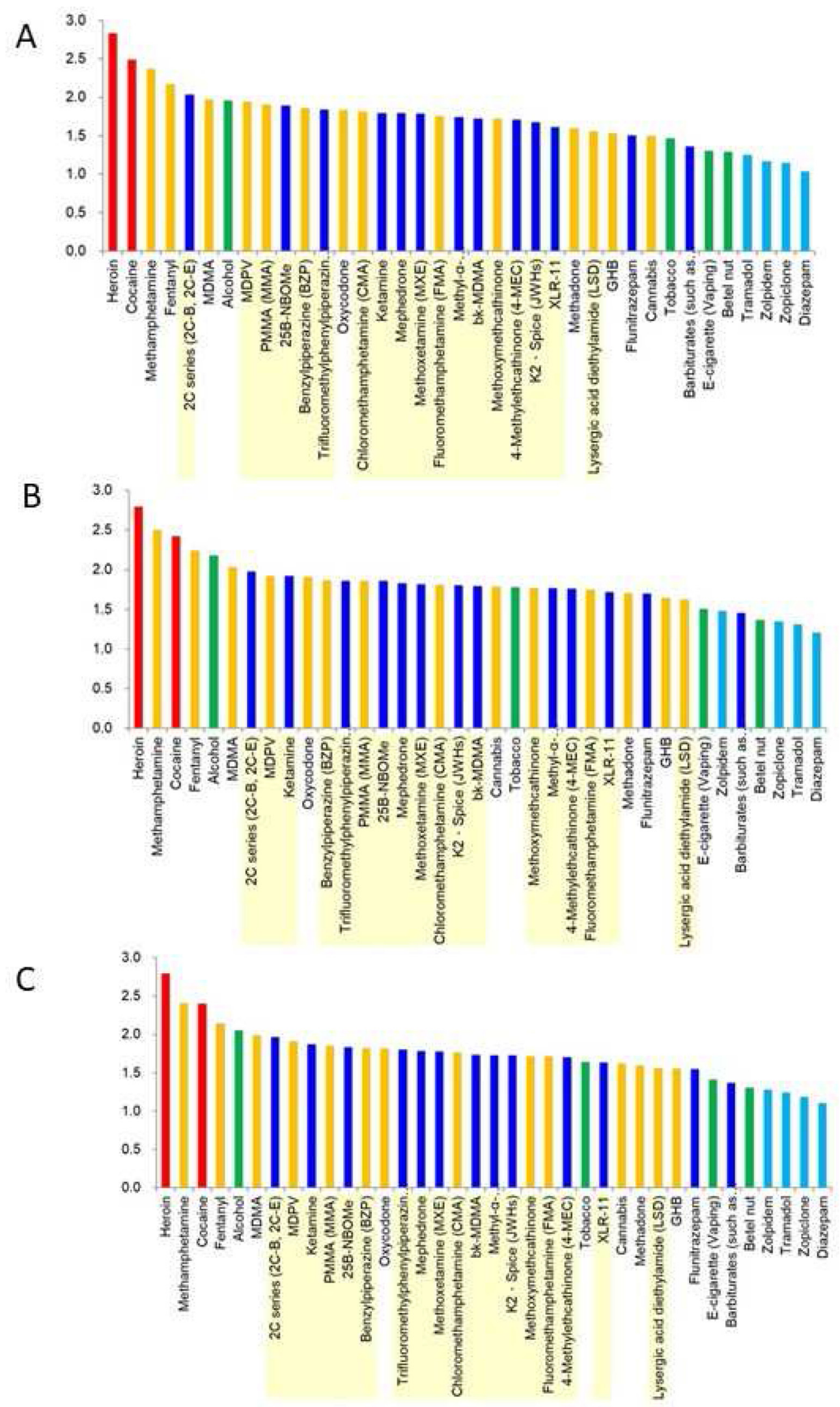

Fig. 4. Rank of mean harm scores for 37 substances based on (A) Nutt's 9 parameters; (B) 8 indicators based on Taiwan's Statute and (C) 11 indicators in this study. 
conduct a linear regression prediction analysis; we selected important impact indicators for these 3 models.

\subsubsection{Model A: Nutt's 9 parameters.}

We analyzed the predictive ability of Nutt's 9 parameters for schedule levels $(\mathrm{Y})$ of substance use by linear regression, selecting 7 indicators with significant prediction capabilities: criminality $\left(X_{\mathrm{a} 1}\right)$, euphoria $\left(X_{a_{2}}\right)$, health-care costs $\left(X_{\mathrm{a} 3}\right)$, acute physical harm $\left(X_{a 4}\right)$, chronic physical harm $\left(X_{a 5}\right)$, withdrawal $\left(\mathrm{X}_{\mathrm{a} 6}\right)$ and international misuse prevalence $\left(X_{a 7}\right)$. he prediction model for estimating the schedule levels of control drug was as follows:

$\mathrm{Y}=0.518 \mathrm{X}_{\mathrm{a} 1}+0.383 \mathrm{X}_{\mathrm{a} 2}-0.372 \mathrm{X}_{\mathrm{a} 3}+0.315 \mathrm{X}_{\mathrm{a} 4}-0.268 \mathrm{X}_{\mathrm{a} 5}-$ $0.111 X_{a 6}+0.101 X_{a 7}$

There was no multicollinearity among these variables; the model was found to have $49.9 \%$ explanatory power.

\subsubsection{Model B: 8 indicators based on Taiwan's} SPCID.

We then analyzed the predictive ability of 8 indicators based on Taiwan's SPCID for schedule levels (Y) of substance use, selecting 6 indicators with significant prediction capabilities: criminality $\left(X_{b 1}\right)$, euphoria $\left(X_{b 2}\right)$, health-care costs $\left(X_{b 3}\right)$, national misuse prevalence $\left(X_{b 4}\right)$, withdrawal $\left(X_{b 5}\right)$ and psychological dependence $\left(X_{b 6}\right)$. The prediction model for estimating the schedule levels of control drug was:

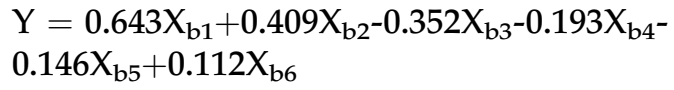

Again, with no multicollinearity, the prediction model was found to have $48.5 \%$ explanatory power.

\subsubsection{Model C: 11 indicators in this study.}

We further analyzed the predictive ability of 11 indicators for schedule levels $(\mathrm{Y})$ of substance use by linear regression and selected the 7 indicators from the first model, but added two, national misuse prevalence and infectious hazard. The prediction model for estimating the schedule levels of control drug was:

$$
\begin{aligned}
& \mathrm{Y}=0.468 \mathrm{X}_{\mathrm{c} 1}+0.353 \mathrm{X}_{\mathrm{c} 2}-0.278 \mathrm{X}_{\mathrm{c} 3}-0.211 \mathrm{X}_{\mathrm{c} 4}+0.288 \mathrm{X}_{\mathrm{c} 5^{-}} \\
& 0.245 \mathrm{X}_{\mathrm{c} 6}+0.144 \mathrm{X}_{\mathrm{c} 7}-0.129 \mathrm{X}_{\mathrm{c} 8}+0.097 \mathrm{X}_{\mathrm{c} 9}
\end{aligned}
$$

With no multicollinearity, the prediction model had $52.4 \%$ explanatory power. Based on this, the predictive explanatory power of model $\mathrm{C}$ was better than the other two models and was explained by the health-care costs, national misuse prevalence, chronic physical harm and withdrawal have positive

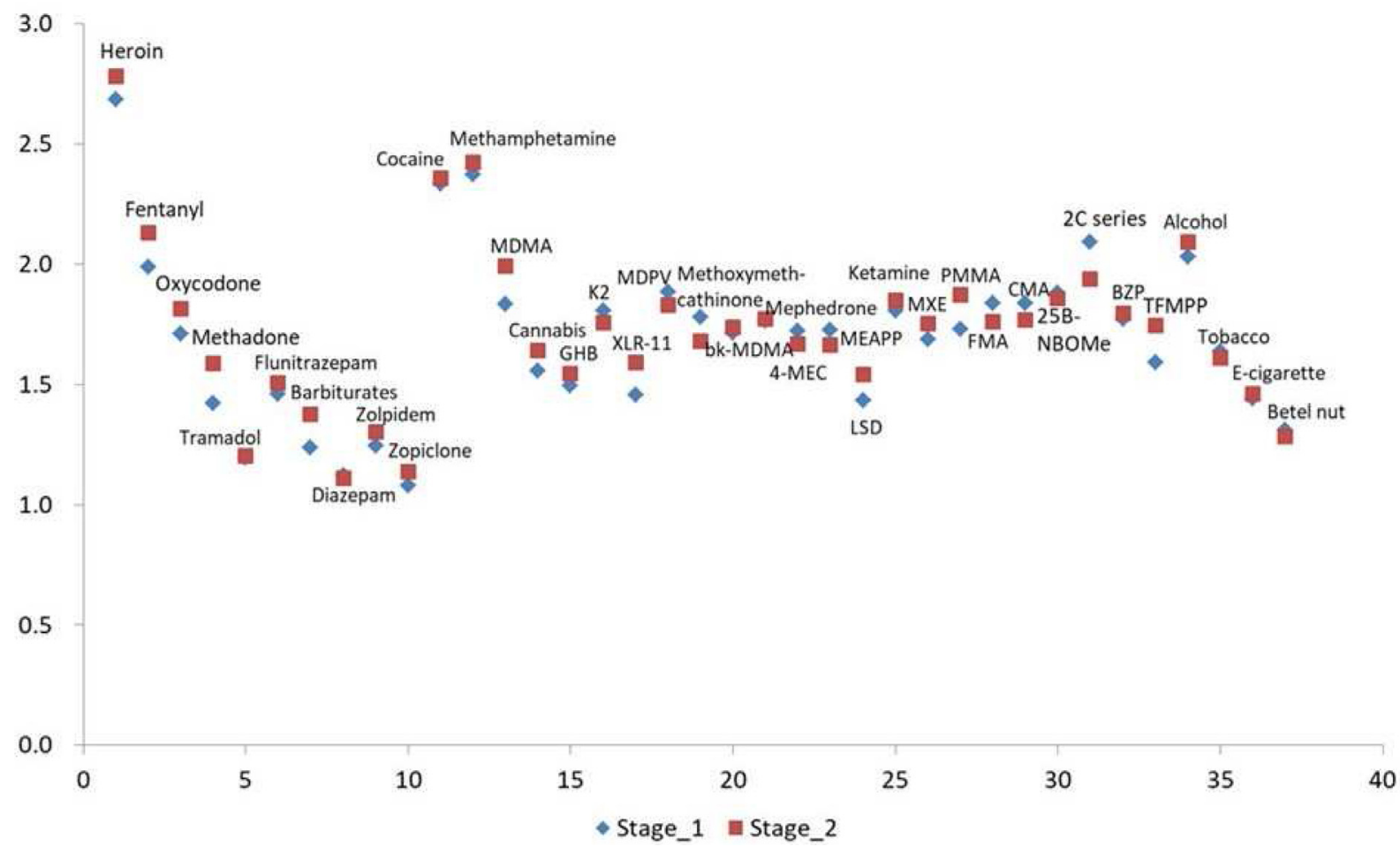

Fig. 5. Comparison of the mean harm scores for 37 substances between stage 1 and stage 2 . 
regression. The national misuse prevalence and international misuse prevalence both were important indicators for harm assessment.

The variance inflation factor (VIF) of each parameter for the prediction model was between 1.674 to 4.432 , indicating that there was no collinearity between parameter and the prediction model. The $\mathrm{p}$-values with $\mathrm{t}$-test between parameters for the prediction models were all less than 0.001 , demonstrating these parameters were predictive. The pvalues of the t-test between two models were less than 0.000 , indicating that there were significant differences between the models. The RMSE (Root mean squared error) of these 3 models was 0.8062 (model A), 0.8167 (model B) and 0.7849 (model C), respectively, showing that the deviation of model $\mathrm{C}$ was the smallest.

\section{Discussion}

Misuse of NPS has posed a global threat to public health but most NPS have not been controlled because of their easy modification on the chemical structures and lack of misuse information. Our previous research demonstrated that Taiwan controlled the least NPS items among the Northeastern countries because of rigid criteria to schedule an NPS item [7, 11]. According to Taiwan's SPCID, a substance cannot be scheduled as a controlled drug unless it possesses all three characteristics: addictive potential, misuse liability and social harm liability. It explains why there were only 52 NPS items scheduled by the end of 2019 in Taiwan as shown in the results. Therefore, it would be a priority to schedule a potentially addictive substance or an NPS item before it becomes an issue.

To rationally schedule a potentially addictive substance, Nutt et al. developed a nine-category of harm model with an expert Delphi procedure [8]. However, this model did not take misuse situation of a potentially addictive substance into consideration. In contrast, the criteria of Taiwan's SPCID did not take the factors of physical harm into account. Thus, in this study, the current situation of drug misuse was first evaluated using epidemiological approaches. From the results of the supply side on drug seizures (Fig. 1, 2-A) and demand side on the scale of drug misuse (Fig. 2-B, 3), we found that ketamine, (meth)amphetamine and heroin have been the most prevalent drugs in Taiwan. While (meth)amphetamine and heroin have been the predominant drugs for several decades, ketamine misuse has not been a problem until the early 2000s $[9,10]$. The NPS other than ketamine, such as synthetic cannabinoids (XLR-11, JWHs); synthetic cathinones (MDPV, methoxymethcathinone, 4MEC, bk-MDMA, mephedrone, MEAPP); phenethylamines (PMMA, FMA, CMA, 2C-B, 2C-E); and piperazines (BZP, TFMPP) have also been on the rise (Fig. 1-B, 2-A and 3).

It should also be noted that the detection capability of drug use from urine samples were limited by the availability of screening tools (such as test kits or assay methods) to screen and/or drug standards to confirm a specific drug. Since many, if not most, NPS have not been scheduled for control, the assay methods or standard preparations for NPS detection are often not available. As a result, the misuse situation of an emerging NPS item may be underestimated.

In reference to the drug misuse situations, the assessment of drug-related harms with an expert Delphi procedure for drug scheduling was conducted. Three models were applied to analyze and rank the harms of 37 substances, including legal addictive substances, traditional drugs and NPS. As shown in Fig. 4-A, Nutt's model did not take the misuse situation into consideration and it may explain why the ranking score of cocaine was higher than that of methamphetamine in the SPCID model. On the other hand, the SPCID model did not take the factors of physical harm into account (Fig. 4-B). As a result, the harm score ranking of PMMA was lower in the SPCID model than that of Nutt's model even though PMMA caused many fatalities in recent years [11]. Therefore, for effective and rational drug scheduling, drug-related harms were integrated from Nutt's model and Taiwan's Statute for the Prevention and Control of Illicit Drugs (SPCID) into four dimensions, namely, addiction, misuse, social harm and physical harm (Table 1). These four dimensions were further divided into 11 indicators and applied to assess harms of the 37 substances. Among the 11 indicators that corresponded to the four dimensions, 7 had significant prediction capabilities.

According to the analysis of the integrated results (Fig. 4-C), heroin posed the highest risk, followed by (meth)amphetamine and ketamine. The harm scores of two schedule III NPS (ketamine and synthetic cathinones) and a legal substance (alcohol) were higher than those of some schedule II drugs (such as cannabis, PCP, GHB and methadone), indicating the harm of some NPS, at least ketamine and synthetic cathinones in this study, may have been underestimated.

From the misuse statistics shown in Fig. 1-B, we could also observe that regulatory intervention would have some impacts on the misuse of NPS as 
evidenced by the quantity of NPS seizures that dropped significantly after regulation. PMMA, regulated as a schedule II drug in 2006, only seized in 2009 at 30.2 grams, and 960.1 grams in 2015, and there were no further seizures. Methoxymethcathinone was regulated as a schedule II drug in 2006 and only 3,240.8 grams were seized at the same year, no further seizures. Misuse of synthetic cathinones such as MDPV, mephedrone, methoxymethcathinone and bk-MDMA deserves further attention. Their seizures had declined after regulation, but were still seized every year. Therefore, an appropriate scheduling system is crucial to effectively control NPS misuse.

The results of LDTU (Fig. 2-A and 2-B) indicated multi-component substances detected in the urine specimens or non-urine specimens have become more prevalent in recent years. Although single addictive substances were still the majority in the positive urine specimens, positive urines detected with more than 5 substances were increasing, especially in 2018 and 2019. One urine specimen even contained more than 15 components. The proportion of non-urine specimens with two components increased from $16.2 \%$ in 2006 to $55.0 \%$ in 2019. In 2014, 23 specimens were tested positive with 16 components, and 11 specimens were tested positive with 15 components in 2016. In 2019, one nonurine specimen was found to contain 15 addictive substances. Multiple drugs in the NPS increase the health risk that cannot be ignored. It is suggested that precautionary measures should be taken for preventive purposes.

In this study, national prevalence of misuse was shown as an important predictor of harm assessment. These 11 indicators of harm assessment of misuse of drugs can help develop a proper scheduling system for the management of controlled/ illicit drugs.

\section{Conclusions}

Use of NPS has been a global concern. The misuse of NPS in Taiwan, initiated in the early 2000s with ketamine misuse, has been extended to include synthetic cathinones, such as mephedrone, MEAPP, bk-MDMA and MDPV; synthetic cannabis, such as XLR-11 and K2 (JHWs); and phenethylamines, such as PMMA and 2C series. So far, only 52 NPS had been scheduled, a number far less than the 950+ NPS defined by the UNODC. It could be due to Taiwan's current rigid criteria in the SPCID addictive potential, abuse liability, and social harm liability - to schedule specific drugs as controlled substances [11].
We have also shown that Taiwan's scheduling principle did not include the physical harm as an assessment indicator, while the research by Nutt et al. ignored the influence of national misuse prevalence. Therefore, based on analysis of the predictive ability of indicators, we integrated the Nutt's and Taiwan's SPCID models into the model C to estimate the drug-associated harm for appropriate scheduling. Addiction, misuse and social hazard were all important factors to assess harms in the model. These indicators can be used for the harm assessment of misused drugs that helps develop a rational and site-specific scheduling system for the management of controlled/illicit drugs.

Proper drug scheduling is the first step in the management of drug use problems. Facing the threats of NPS misuse, it is imperative to implement a rational and effective scheduling system for appropriate management. This study provides a mechanism to scrutinize, and hopefully to improve, the current evaluation process for drug scheduling.

\section{Conflict of interest}

The authors declare no conflict of interests.

\section{Acknowledgements}

The authors would like to thank that supported in part by grants from Food and Drug Administration, Ministry of Health and Welfare, Taiwan (MOHW103-FDA-61301); University of Florida Substance Abuse Training Center in Public Health (DA-T32-035167-06); National Drug Early Warning System (NDEWS) Coordinating Center (U01-DA051126-01); Identifying Patterns of Human Polysubstance Use to Guide Development of Rodent Models(R21-DA-045140-03).

\section{References}

[1] United Nations Office on Drugs and Crime. The challenge of new psychoactive substances. March 2013.

[2] United Nations Office on Drugs and Crime. Global Synthetic Drugs Assessment: Amphetamine-type stimulants and new psychoactive substances. 2014.

[3] United Nations. Single Convention on Narcotic Drugs, United Nations. 1961.

[4] United Nations. Convention on Psychotropic Substances, United Nations. 1971.

[5] United Nations Office on Drugs and Crime. Categories of new psychoactive substances sold in the market.

[6] United Nations Office on Drugs and Crime. World Drug Report 2019; Vienna. June 2019.

[7] Feng LY, Wada K, Chung H, Han E, Li JH. Comparison of legislative management for new psychoactive substances control among Taiwan, South Korea, and Japan. Kaohsiung J Med Sci 2020;36:135-42.

[8] Nutt D, King LA, Saulsbury W, et al. Development of a rational scale to assess the harm of drugs of potential misuse. Lancet 2007;369:1047-53. 
[9] Yu WJ, Tsay WI, Li JH. Current status of substance abuse and HIV in Taiwan. J Food Drug Anal 2013;21(4):s27-32.

[10] Li JH, Vicknasingam B, Cheung YW, et al. To use or not to use: an update on licit and illicit ketamine use. Subst Abuse Rehabil 2011;2:11-20.

[11] Feng LY, Li JH. New Psychoactive Substances in Taiwan: Challenges and Strategies. Current Opinion in Psychiatry 2020;33(4):306-11.

[12] Taiwan FDA. Drug abuse cases and test statistics data from 2006 to 2019.
[13] Wang S, Yu L, Tang L, Wang SY. A novel seasonal decomposition based least squares support vector regression ensemble learning approach for hydropower consumption forecasting in China. Energy 2011;36(11):6542-54.

[14] Yu L, Zhao Y, Tang L, Yang Z. Online big data-driven oil consumption forecasting with Google trends. International Journal of Forecasting 2019;35:213-23.

[15] Taiwan FDA. National Substance Use Survey. 2014. 